\title{
Estimation of Non-stationary Process Variance in Multistage Manufacturing Processes Using a Model-based Observer
}

\author{
Ester Sales-Setién, Ignacio Peñarrocha-Alós, and José V. Abellán-Nebot
}

\begin{abstract}
In this work we propose a recursive algorithm to estimate the process variance in multistage manufacturing or assembly processes. We use a replicated model that includes the process variance to be estimated as a time-varying state that changes slowly. For this model, we develop an estimation strategy including tuning parameters that play a direct role in the trade-off between the estimation accuracy and the adaptation to changes. We also develop a statistical confidence interval for the estimations which enhances the decision of whether the process variances have changed. Unlike other batch methods in the literature, our proposal is computed recursively, and it allows us to tune the trade-off between the convergence speed and the accuracy without modifying the sample size, which only contains the data of the last manufactured piece.
\end{abstract}

Note to Practitioners-Variation reduction appears as the key to succeed in improving product quality, which is a major asset in manufacturing. To achieve variation reduction, it is necessary to quickly identify the root causes which are responsible for these variations. The state of the art in root cause analysis is based on a stationary point of view, where the estimations regarding the mean and variance of the variation sources are based on the sample provided by a batch of multiple manufactured pieces. This paper focuses on the development of an online estimation strategy which recursively updates the estimation of the variance of the variation sources with the data provided by the last manufactured piece in order to enhance a rapid decision of whether any fault has occurred in the process at the same time of reducing the computational cost and the data storage needs. We present a design strategy of the estimation algorithm which sets the tradeoff between the accuracy of the estimations and their capability of tracking changes through two well-known physically-meaningful engineering parameters: the cumulative squared error under abrupt changes and the variance of the estimations. Two detailed cases of study illustrate the effectiveness of this method. The strategy presented in this paper represents a novel approach for dealing with quality-control online algorithms and it can be used for quality improvement of multistage manufacturing processes.

Index Terms-Process variance estimation, model-based observer, multistage manufacturing process, variation propagation.

\section{INTRODUCTION}

Nowadays, the highly competitive markets demand customized and high quality products with minimum lead times exerting a great pressure on companies to optimize their

This work was supported in part by MECD under Grant FPU14/01592 and by projects P1-1B2015-42 and P1-1B2015-53 from Universitat Jaume I of Castelló and TEC2015-69155-R from MINECO.

E. Sales-Setién, I. Peñarrocha-Alós and J.V. Abellán-Nebot are with the Department of Industrial Engineering and Design, Universitat Jaume I, Castellón, Spain. resources throughout the product development cycle. At the manufacturing stage, process engineers are focused on the analysis and reduction of process variation to reduce cost, improve product quality and minimize production ramp-up times when new products are launched. However, process variation reduction throughout the manufacturing process is a challenging task, especially in complex processes such as multistage manufacturing processes (MMPs). These processes are those that produce products under multiple setups where the operations conducted at the first stages have an influence on the manufacturing operations down-streams [1]. For instance, the process assembly of automobile bodies is a MMP composed of a series of single stages where, at each stage, the body from previous stages is held in a work-holding structure to assemble new components and, after the welding operation, the body moves forward to the next stages in order to complete additional assembly operations. In MMPs, statistical process control (SPC) techniques are commonly applied to control the manufacturing process over time by monitoring the quality characteristics of the product. The statistical control charts applied in SPC are effective tools to detect process changes and ensure product quality. However, the identification of root causes that may be inferred from charting results would be significantly limited if the interrelationships between the process variables and the key product characteristics (KPCs) are not explicitly modelled. To overcome this limitation, $\mathrm{Li}$ et al. [2] proposed a causation-based $T^{2}$ decomposition method where the causal relationships among variables are modelled by a Bayesian network. The interpretation and decomposition of Hotelling's $T^{2}$ together with the causal information of the process and the KPCs variables let the proposed SPC method to trace backward from certain quality problems to the variables that are the root causes. An improvement of this method is provided in Verron et al. [3] where the decompositions are computed within the Bayesian network itself.

The state-space model from control theory has also been successfully applied to model the relationships between variation sources and product dimensional quality measurements in MMPs [4]-[11]. The use of the state-space model with SPC techniques for root cause identification was proposed in Zou and Tsung [12]. In their work, a multivariate exponential weighted moving average scheme with the generalized likelihood test that fully incorporates directional information based on the state-space model was proved to be an effective solution for process monitoring and fault diagnosis. Besides these SPC techniques, different variation source identication 
techniques such as those based on MLE estimators, REML estimators and MINQUE estimators have been proposed in MMPs using the state-space model as a linear mixed model. In [13], the application of MLE and MINQUE estimators in MMPs was discussed for estimating both the mean and the variance of the process variation sources. A hypothesistesting procedure was also developed to provide confidence level of each of the estimation results which let the plant engineers determine if process faults regarding the mean and the variance of the process variation sources exist in terms of statistical significance. However, these methods have been mainly applied in biological and agricultural fields [14] and they are primarily applicable to offline experiments where the sample size is small and the computation time is not a concern. In MMP, large quantity of data may be available and the detection and identification of any process malfunctioning need to be carried out faster and efficiently.

For this reason, online estimators with low computation cost and fast response have been investigated in previous researches proving their applicability. A good comparison of online estimators of the variance of the process variation sources (i.e., process variance estimators) and their performance can be found in [15], where the process variance estimators from the research works in [16]-[19] were analyzed. These estimators entail closed-form expressions and are more cost-effective than the MLE method, particularly for large sample sizes, and are thus more suitable for online quality control. However, the estimators analyzed are only adequate when the MMP is stationary since the estimations are based on the sample of data of the last $N$ pieces and only when most of the $N$ pieces are affected by the process faults the estimators can correctly detect and quantify the process variance changes. Therefore, these estimators present a delay in identifying the variation sources which negatively impacts on the efficiency of online quality control actions. For instance, a certain number of parts may be manufactured before the variation source is identified which compromises the quality in terms of variance of the final product and produces an increase of non-quality costs. To overcome this limitation the application of non-stationary process variance estimators in MMPs has to be investigated.

This paper discusses the use of a model-based observer that recursively updates the estimated process variance with the measurements from each new processed piece. The observer demands few computational burden and it has an asymptotic behavior. We also discuss how to tune the observer for fixing the trade-off between the convergence speed under process variance changes and the steady-state error for stationary process variances.

This paper is organized as follows. The problem statement is presented in Section II. Section III presents the proposed approach for modeling the non-stationary behavior of the process variances in MMPs that enables the application of observer-based strategies for variation source identification. Section IV explains the estimation procedure of the process variance and it details a design strategy of the observer for ensuring a specific performance of the estimator. In Section $\mathrm{V}$ we include the design of confidence intervals for the estimates so as to enhance statistical hypothesis testing for

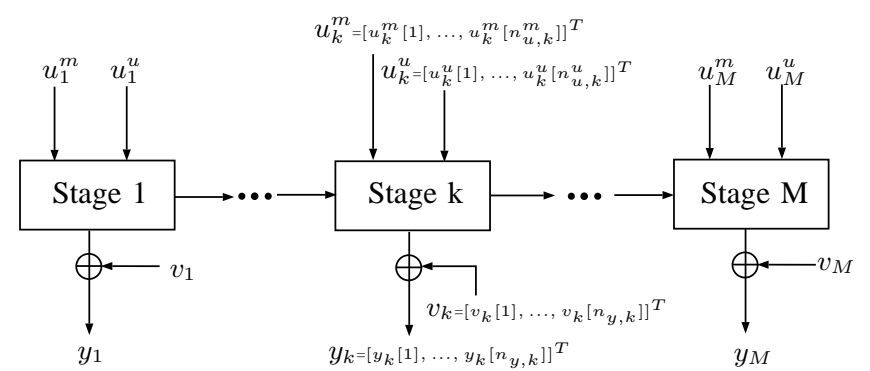

Figure 1. Diagram of a MMP

fault diagnosis. Section VI briefly compares the proposed estimates with offline existing estimators and Section VII presents a case of study to validate the approach. Finally, Section VIII summarizes the main conclusions of the paper.

Notation: Let $M \in \mathbb{R}^{n \times n}$ be a square matrix and $m \in \mathbb{R}^{n}$ be some vector. $M[i, j]$ denotes the element in the $i$-th row and $j$-th column of $M$ and $m[i]$ denotes the $i$-th element in $m . I_{n} \in \mathbb{R}^{n \times n}$ is the identity matrix of size $n$ and $\mathbf{1}_{n} \in \mathbb{R}^{n}$ is the unitary column vector of size $n$. $\operatorname{vec}(M) \in \mathbb{R}^{n^{2}}$ denotes the vectorization of $M$ and $\operatorname{vec}^{-1}(\operatorname{vec}(M))=M$. $\operatorname{diag}(M) \in \mathbb{R}^{n}$ is the operator that returns a column vector with the diagonal entries of $M . \operatorname{diag}^{-1}(\operatorname{diag}(M))$ is a diagonal matrix with the elements $M[i, i]$ in its diagonal. Product is denoted as $\Pi$, summation is denoted as $\sum$ and the direct sum is denoted as $\bigoplus$, so that $\bigoplus_{i} m[i] \in \mathbb{R}^{n \times n}$ is a diagonal matrix containing the elements of $m$ in its diagonal. $M^{\otimes^{2}}$, and $M^{\circ 2}$ represent the Kronecker and Hadamard product of $M$ and $M$ (i.e., $M^{\otimes^{2}}=M \otimes M$ and $M^{\circ 2}=M \circ M$ ). Expected value and probability are denoted as $\mathbf{E}\{\cdot\}$ and $\operatorname{Pr}\{\cdot\}$.

\section{Problem Statement}

In a MMP the deviations caused by the variation sources of each stage propagate along the production line. At certain stage the dimensional variability of a piece consists of two components: one derived from the previous stages and another created at the current stage. Since these deviations are much smaller than their corresponding nominal value, they can be represented by a linear state-space model as

$$
\begin{aligned}
& x_{k}(i)=A_{k-1} x_{k-1}(i)+B_{k} u_{k}^{m}(i)+u_{k}^{u}(i), \\
& y_{k}(i)=C_{k} x_{k}(i)+v_{k}(i),
\end{aligned}
$$

where $x_{k}(i) \in \mathbb{R}^{n_{x, k}}$ represents the dimensional variability of piece $i=\{1, \ldots, \infty\}$ at stage $k=\{1, \ldots, M\}$. Vector

$$
u_{k}(i)=\left[\begin{array}{l}
u_{k}^{m}(i) \\
u_{k}^{u}(i)
\end{array}\right] \in \mathbb{R}^{n_{u, k}}
$$

with $n_{u, k}=n_{u, k}^{m}+n_{u, k}^{u}$ and $n_{u, k}^{u}=n_{x, k}$ is the input vector including both the modeled and unmodeled variation sources of the $k$-th stage affecting the $i$-th piece. Vector $y_{k}(i) \in \mathbb{R}^{n_{y, k}}$ is the output vector denoting the measurements of the $i$-th piece at the $k$-th stage and $v_{k}(i) \in \mathbb{R}^{n_{y, k}}$ takes account of the corresponding measurement noises. This kind of stage and piece indexed state-space models, depicted in Fig.1, has been extensively used in MMP applications as shown in [4]-[10], which are referred for further modeling details. 
As proposed by Apley and Shi [16], model (1) can be algebraically transformed into a linear replicated model. Setting the initial conditions to 0 , it yields

$$
y(i)=\Gamma u(i)+v(i),
$$

where $y(i), u(i)$ and $v(i)$ stand, respectively, for all the measurements, inputs and noises that affect each piece $i$ throughout the MMP, i.e.,

$$
y(i)=\left[\begin{array}{c}
y_{1}(i) \\
\vdots \\
y_{M}(i)
\end{array}\right], u(i)=\left[\begin{array}{c}
u_{1}(i) \\
\vdots \\
u_{M}(i)
\end{array}\right], v(i)=\left[\begin{array}{c}
v_{1}(i) \\
\vdots \\
v_{M}(i)
\end{array}\right]
$$

with $y(i), v(i) \in \mathbb{R}^{n_{y}}, n_{y}=\sum_{k} n_{y, k}$ and $u(i) \in \mathbb{R}^{n_{u}}, n_{u}=$ $\sum_{k} n_{u, k}$. Matrix $\Gamma$ can be obtained from $A_{k}, B_{k}$ and $C_{k}$ as explained in the references.

Many works as [15] consider that (3) describes a stationary process. For process variance estimation purposes, it is then possible to establish the following relation:

$$
\boldsymbol{\Sigma}_{y}=\Gamma \boldsymbol{\Sigma}_{u} \Gamma^{T}+\boldsymbol{\Sigma}_{v},
$$

where

$$
\begin{aligned}
\boldsymbol{\Sigma}_{y} & =\mathbf{E}\left\{y y^{T}\right\}, \\
\boldsymbol{\Sigma}_{u} & =\mathbf{E}\left\{u u^{T}\right\}, \\
\boldsymbol{\Sigma}_{v} & =\mathbf{E}\left\{v v^{T}\right\} .
\end{aligned}
$$

However, the process variances in (5) may change its value at the moment when certain piece $i$ is manufactured. This is due to different effects such as the wear and tear to which the MMP is prone. Then, we can no longer consider the MMP as a stationary process and we rewrite (5) as

$$
\boldsymbol{\Sigma}_{y}(i)=\Gamma \boldsymbol{\Sigma}_{u}(i) \Gamma^{T}+\boldsymbol{\Sigma}_{v}(i),
$$

where $\boldsymbol{\Sigma}_{y}(i), \boldsymbol{\Sigma}_{u}(i)$ and $\boldsymbol{\Sigma}_{v}(i)$ denote the covariance of the measurements, the variation sources and the measurements noises at the moment when certain piece $i$ is processed, i.e.,

$$
\begin{aligned}
& \boldsymbol{\Sigma}_{y}(i)=\mathbf{E}\left\{y(i) y(i)^{T}\right\}, \\
& \boldsymbol{\Sigma}_{u}(i)=\mathbf{E}\left\{u(i) u(i)^{T}\right\}, \\
& \boldsymbol{\Sigma}_{v}(i)=\mathbf{E}\left\{v(i) v(i)^{T}\right\} .
\end{aligned}
$$

Remark 1. The variables $\boldsymbol{\Sigma}_{y}(i), \boldsymbol{\Sigma}_{u}(i)$ and $\boldsymbol{\Sigma}_{v}(i)$ defined in (8) are time-varying stochastic variances [20], [21] and they represent the variance of $y, u$ and $v$ for a population of pieces manufactured at the manufacturing conditions of the $i$-th piece.

Taking [15] as reference, in this work we assume that:

- The underlying distributions of $u$ and $v$ are Gaussian.

- The input vector $u$ is zero-mean since it represents the deviation from the designed nominal position. The variation sources are independent, so that $u$ has a diagonal non-stationary covariance matrix, i.e.,

$$
\boldsymbol{\Sigma}_{u}(i)=\left[\begin{array}{ccc}
\sigma_{u}^{2}(i)[1] & & 0 \\
& \ddots & \\
0 & & \sigma_{u}^{2}(i)\left[n_{u}\right]
\end{array}\right],
$$

where $\sigma_{u}^{2}(i)[p]$ represents the dimensional variance of the $p$-th variation source of the MMP, i.e., $p=\left\{1, \ldots, n_{u}\right\}$, at the moment where the $i$-th piece is manufactured.

- The noise vector $v$ is zero-mean, independent of $u$ and it has a diagonal non-stationary covariance matrix,

$$
\boldsymbol{\Sigma}_{v}(i)\left[\begin{array}{ccc}
\sigma_{v}^{2}(i)[1] & & 0 \\
& \ddots & \\
0 & & \sigma_{v}^{2}(i)\left[n_{y}\right]
\end{array}\right],
$$

where $\sigma_{v}^{2}(i)[r]$ represents the dimensional variance of the noise of the $r$-th sensor of the process, i.e., $r=$ $\left\{1, \ldots, n_{y}\right\}$, at the moment where the $i$-th piece is manufactured.

- As many works in the field (e.g., [15] ), we assume that all the measurement noises have the same variance. Then, we rewrite $\boldsymbol{\Sigma}_{v}(i)$ as

$$
\boldsymbol{\Sigma}_{v}(i)=\sigma_{v}^{2}(i) I_{n_{y}}
$$

with $\sigma_{v}^{2}(i)$ an scalar denoting this shared process variance. It is straightforward to extend the results of this work to the case where the sensor noises are characterized by different variances ${ }^{1}$.

For the sake of readability, we denote the components of $\boldsymbol{\Sigma}_{u}(i)$ and $\boldsymbol{\Sigma}_{v}(i)$ as $\sigma^{2}(i)[j]$ with $j=\left\{1 \ldots n_{j}\right\}$ and $n_{j}=n_{u}+1$, i.e.,

$$
\sigma^{2}(i)[j] \equiv \begin{cases}\sigma_{u}^{2}(i)[j], & j<n_{j}, \\ \sigma_{v}^{2}(i), & j=n_{j} .\end{cases}
$$

The stationary process variance estimator as the ones studied in [15] (i.e., the Least-Squares fit estimator (LSE), the estimator in Apley and Shi (ASE) [16], the estimator in Ding, Shi and Ceglarek (DSCE) [18] and the estimator presented in Stoica and Nehorai (SNE) [17]) use the sampling variance of a batch of $N$ pieces, i.e.,

$$
S_{y}=\frac{\sum_{i=1}^{N} y(i) y(i)^{T}}{N},
$$

as an estimate of $\boldsymbol{\Sigma}_{y}$ in (5). For the study of a non-stationary process, we could propose to infer a time-varying population variance every time that a new piece $i$ is manufactured. If we used the sample provided by the batch of the last $N$ pieces, an estimation of $\boldsymbol{\Sigma}_{y}(i)$ in (7) would be given by

$$
S_{y}(i)=\frac{\sum_{\iota=i-N}^{i} y(\iota) y(\iota)^{T}}{N} .
$$

The use of (14) is computationally costly since a large sample size (i.e., the data provided by a batch of $N$ pieces) is used for guaranteeing accurate estimates of the process variances $\sigma^{2}(i)[j]$ and no individual control over the accuracy of each process variance estimate is available. For its part, the use of a sample of large size in (13) for ensuring accurate estimates delays the estimation of variance changes and thus the decision of whether the process has experienced faults or not. In this

\footnotetext{
${ }^{1}$ It is well known that the maximum number of different process variances which can be estimated depends on the structural properties of the MMP under consideration. See [22], [23] for studying the diagnosability issues of each case.
} 
work, we propose an alternative strategy for identifying the components of $\boldsymbol{\Sigma}_{u}(i)$ and $\boldsymbol{\Sigma}_{v}(i)$ and their possible changes taking into account

- the accuracy of the estimations,

- the time needed to track their changes,

- the computational cost,

- the data storage,

- the design flexibility.

To fulfill these requirements we develop a model for MMPs which includes the non-stationary behavior of the process variances $\sigma^{2}(i)[j]$ and enables the application of observerbased strategies. These strategies allow us to incrementally improve the accuracy of the estimates $\hat{\sigma}^{2}(i)[j]$ with the data provided by each new piece without the need of storing or using the data of the previous pieces in the computation.

\section{VARIATION PROPAGATION MODEL}

In order to develop a model for MMPs with non-stationary process variances of the variation sources and noises we exploit the Gaussian nature of $u(i)$ and $v(i)$ in (3). Let us first introduce the following lemma.

Lemma 1. ([24]) Let $g(i) \in \mathbb{R}^{n_{g}}$ be a vector of zero-mean independent Gaussian noises, i.e.,

$$
g(i) \sim \mathcal{N}\left(0, \boldsymbol{\Sigma}_{g}(i)\right)
$$

with $\boldsymbol{\Sigma}_{g}(i)$ certain diagonal and varying covariance. If $g_{u}(i)$ denotes the vector including $n_{g}$ zero-mean independent Gaussian noises of unitary covariance, i.e.,

$$
g_{u}(i) \sim \mathcal{N}\left(0, I_{n_{g}}\right),
$$

vector $g^{\prime}(i)$ defined as

$$
g^{\prime}(i)=\boldsymbol{\Sigma}_{g}(i)^{\frac{1}{2}} g_{u}(i)
$$

is distributed as (15).

Applying Lemma 1, the random signals $u(i)$ and $v(i)$ in (3) can be rewritten as

$$
u(i)=D(i) \eta(i), \quad v(i)=R(i) \nu(i) .
$$

where

$$
\eta(i)=\left[\begin{array}{c}
\eta(i)[1] \\
\vdots \\
\eta(i)\left[n_{u}\right]
\end{array}\right], \nu(i)=\left[\begin{array}{c}
\nu(i)[1] \\
\vdots \\
\nu(i)\left[n_{y}\right]
\end{array}\right]
$$

are column vectors containing independent zero-mean gaussian sequences with unitary time-invariant covariances, i.e.,

$$
\begin{gathered}
\mathbf{E}\{\eta(i)\}=0_{n_{u}}, \mathbf{E}\left\{\eta(i) \eta(i)^{T}\right\}=I_{n_{u}}, \\
\mathbf{E}\{\nu(i)\}=0_{n_{y}}, \mathbf{E}\left\{\nu(i) \nu(i)^{T}\right\}=I_{n_{y}}, \\
\mathbf{E}\left\{\eta(i) \nu(i)^{T}\right\}=0_{n_{u} \times n_{y}} .
\end{gathered}
$$

Matrix $D(i)$ contains the standard deviation of the components of $u(i)$, i.e.,

$$
D(i)=\left[\begin{array}{ccc}
\sigma_{u}(i)[1] & & 0 \\
& \ddots & \\
0 & & \sigma_{u}(i)\left[n_{u}\right]
\end{array}\right],
$$

and fulfills

$$
D(i) D(i)^{T}=\boldsymbol{\Sigma}_{u}(i) \text {. }
$$

The same applies to matrix $R(i)$ w.r.t. $v(i)$ (i.e., $R(i)=$ $\left.\sigma_{v}(i) I_{n_{y}}\right)$. Note that signals $\eta(i)$ and $\nu(i)$ represent the Gaussian variations in $u(i)$ and $v(i)$ while $D(i)$ and $R(i)$ describe the size of these variations. Applying the decomposition in (18), the linear replicated model (3) results in

$$
y(i)=\left[\begin{array}{ll}
\Gamma D(i) & R(i)
\end{array}\right]\left[\begin{array}{l}
\eta(i) \\
\nu(i)
\end{array}\right] .
$$

Defining matrices

$$
\Psi(i)=\left[\begin{array}{ll}
\Gamma D(i) & R(i)
\end{array}\right], \zeta(i)=\left[\begin{array}{l}
\eta(i) \\
\nu(i)
\end{array}\right]
$$

we rewrite $(23)$ as

$$
y(i)=\Psi(i) \zeta(i)
$$

For process variance estimation, the information of interest resides in the diagonal terms of the quadratic expression of (23). Let us then define the output vector $m(i) \in \mathbb{R}^{n_{y}}$ as

$$
m(i)=y(i) \circ y(i) \equiv \operatorname{diag}\left\{y(i) y(i)^{T}\right\},
$$

verifying

$$
m(i)=\operatorname{diag}\left\{\Psi(i) \zeta(i) \zeta(i)^{T} \Psi(i)^{T}\right\} .
$$

Note that $m(i)$ depends on the process variances that we want to estimate. If we define $q(i)$ as the column vector stacking these variances, i.e.,

$$
q(i)=\left[\begin{array}{c}
\sigma^{2}(i)[1] \\
\vdots \\
\sigma^{2}(i)\left[n_{j}\right]
\end{array}\right] \in \mathbb{R}^{n_{j}},
$$

being $\sigma(i)[j]$ the elements of $D(i)$ and $R(i)$ and, thus, included in $\Psi(i)$, the expected value of $m(i)$ satisfies

$$
\mathbf{E}\{m(i)\}=H q(i),
$$

with

$$
H=\left[\begin{array}{ll}
\Gamma^{\circ 2} & \mathbf{1}_{n_{y}}
\end{array}\right] \in \mathbb{R}^{n_{y} \times n_{j}},
$$

where we have taken into account that $\mathbf{E}\left\{\zeta(i) \zeta(i)^{T}\right\}=$ $I_{n_{u}+n_{y}}$ (see Appendix A).

For modeling the non-stationary behavior of the process variances, we propose the following dynamics of the state vector $q(i)$ :

$$
q(i)=q(i-1)+\Delta q(i-1)
$$

where

$$
\Delta q(i)=\left[\begin{array}{c}
\Delta \sigma^{2}(i)[1] \\
\vdots \\
\Delta \sigma^{2}(i)\left[n_{j}\right]
\end{array}\right] \in \mathbb{R}^{n_{j}}
$$

takes account of the process variance differences between two consecutive pieces $i$ and $i+1$. Dynamics of the form of (31) have been widely used in the literature to analyze the behavior of estimation algorithms for non-stationary processes. Note that equation (31) allows modeling every kind of change experienced by the variables $q(i)[j]$ provided an appropriate 
form of the signals $\Delta q(i)[j]$. For instance, abrupt changes in $q(i)[j]$ are produced by impulse signals $\Delta q(i)[j]$ (i.e., $\Delta q(i)[j]$ is only nonzero at the time of the fault appearance), drift changes in $q(i)[j]$ are produced by step signals $\Delta q(i)[j]$ $(\Delta q(i)[j]$ takes a constant value during the fault existence) and parabolic changes in $q(i)[j]$ are produced by ramp signals $\Delta q(i)[j]$.

Thus, the estimations provided by an estimator which takes equation (31) into account would vary in value when any kind of fault form appears in the system. However, such estimator only guarantees estimations with zero-mean steady-state errors when abrupt changes affect the system [25]-[27]. Thus, the estimations are unbiased when no changes occur and when abrupt changes affect the system. If more general changes occurred, the steady-state estimation errors would not be zeromean. For instance, if drift changes took place the steadystate estimation errors would be constant [28]). In any case, however, the estimations would vary in value and the changes experienced by the variables $q(i)[j]$ would be detected.

Remark 2. If zero-mean estimation errors were required for changes beyond abrupt deviations, a more general model in the form of

$$
\begin{aligned}
& \xi(i)=A_{Q} \xi(i-1)+B_{Q} \Delta q(i-1), \\
& q(i)=C_{Q} \xi(i)+D_{Q} \Delta q(i),
\end{aligned}
$$

should be considered. In (33), $\xi(i) \in \mathbb{R}^{n_{\zeta}}$ is an auxiliary state vector of an appropriate dimension so that the dynamics of the forecasted changes can be produced through some matrices $\left(A_{Q}, B_{Q}, C_{Q}, D_{Q}\right)$ and impulse signals $\Delta q(i)$. See [28]-[30] for details on the derivation of these models.

\section{ESTIMATION OF PROCESS VARIANCE}

\section{A. Model-based observer}

For achieving non-stationary process variance estimation, we use a model-based observer. Based on the model defined by equations (27)-(31), we set up the following estimation algorithm. First, we define $\hat{q}(i)$ as the vector that contains the estimation of $q(i)$, i.e., the process variance estimation. Second, we obtain $m(i)$ with the acquired measurements for piece $i$ using expression (26). Then, we estimate $m(i)$ using the last estimated process variance $\hat{q}(i-1)$ and the expression of its expected value given by (29), which involves the model information $H$ :

$$
\hat{m}(i)=H \hat{q}(i-1) .
$$

Finally, we update the process variance estimation with the difference between the measured and estimated output through

$$
\hat{q}(i)=\hat{q}(i-1)+L(i)(m(i)-\hat{m}(i)),
$$

where $L(i)$ is the updating gain matrix which defines the weight between the output prediction error and the last estimated process variance. We define the state estimation error as $\tilde{q}(i)=q(i)-\hat{q}(i)$ and its dynamics is given by

$$
\tilde{q}(i)=\tilde{q}(i-1)+\Delta q(i-1)-L(i)(m(i)-H \hat{q}(i-1)) .
$$

If we add and subtract $H q(i)$ to the difference $m(i)-H \hat{q}(i-1)$ in (36), the dynamics of $\tilde{q}(i)$ can be expressed as

$$
\tilde{q}(i)=\left(I_{n_{j}}-L(i) H\right)(\tilde{q}(i-1)+\Delta q(i-1))-L(i) t(i),
$$

with

$$
t(i)=m(i)-H q(i)
$$

a zero-mean random variable as it derives from the difference between $m(i)$ and its expected value.

Remark 3. Notice that an estimate $\hat{q}(i)$ may be negative for some piece $i$ (i.e., $\hat{q}(i)<0$ ). Provided that $q(i)$ is a vector stacking variance values and it cannot be negative, we postprocess $\hat{q}(i)$ so as to provide a refined estimate of $q(i)$, which we denote as $\hat{q}_{p}(i)$ :

$$
\hat{q}_{p}(i)=\left\{\begin{array}{ll}
\hat{q}(i) & \text { if } \hat{q}(i) \geq 0 \\
0 & \text { otherwise }
\end{array} .\right.
$$

\section{B. Observer Design}

In this section, we propose a design of the gain $L(i)$ of the estimation algorithm (35). Note that the dynamics in (37) can be seen as the estimation error dynamics which is achieved when applying a state observer to a linear system with $\tilde{q}(i)$ the state estimation error, $\Delta q(i)$ the process noise, $t(i)$ the measurement noise, $I_{n_{j}}$ the state matrix and $H$ the output matrix. As the considered process noise $t(i)$ depends on the state, the error dynamics is not linear and simple designs with a constant gain $L(i)$ (as pole placement techniques or stationary Kalman filter approaches) cannot be applied. We then propose a Kalman filter that takes account of the dependence of the noise $t(i)$ on the state which is being estimated (see Appendix B and references [31], [32] for derivation details). The Kalman gain for observer (35) can be computed as

$$
\begin{aligned}
\bar{P}(i) & =\hat{P}(i-1)+Q(i-1), \\
L(i) & =\bar{P}(i) H^{T}\left(H \bar{P}(i) H^{T}+T(i)\right)^{-1}, \\
\hat{P}(i) & =\left(I_{n_{j}}-L(i) H\right) \bar{P}(i),
\end{aligned}
$$

with

$$
\begin{aligned}
Q(i) & =\mathbf{E}\left\{\Delta q(i) \Delta q(i)^{T}\right\}, \\
T(i) & =\mathbf{E}\left\{t(i) t(i)^{T}\right\},
\end{aligned}
$$

and where $\hat{P}(i)$ represents the expected covariance of the state estimation error, i.e.,

$$
\hat{P}(i)=\mathbf{E}\left\{\tilde{q}(i) \tilde{q}(i)^{T}\right\} .
$$

Remark 4. A necessary condition for the stability of observer (35) is that the pair $\left(I_{n_{j}}, H\right)$ is observable, which means that the process variances considered in $q(i)$ are diagnosable. This condition is verified whenever the output matrix $H$ verifies

$$
\operatorname{rank}\{H\} \geq n_{j} .
$$

For computing the covariances $Q(i)$ and $T(i)$ involved in (40), the following considerations should be taken into account. 
- Operating algebraically and taking account of the Gaussian nature and the statistical properties (20) of the noises $\zeta(i)$, one gets that the covariance matrix $T(i)$ is given by

$$
T(i)=\left(\Psi(i) \Psi(i)^{T}\right)^{\circ 2} .
$$

Relation (44) shows the dependence of $T(i)$ on the values $\sigma(i)[j]$ and, thus, on the state vector $q(i)$, which is to be estimated. Provided the slow-varying character of $q(i)$, we have that, in general, $T(i) \simeq T(i-1)$. Then, we propose to approximate covariance $T(i)$ of a piece $i$ by its predicted value from piece $i-1$, i.e.,

$$
T(i) \approx \hat{T}(i-1) .
$$

Matrix $\hat{T}(i-1)$ is computed through (44) with the values in the available postprocessed estimated vector $\hat{q}_{p}(i-1)$.

- The covariance matrix $Q(i)$ is unknown and it can be seen as a multivariate tuning parameter that fixes the performance of the observer. If matrix $Q(i)$ is chosen to be diagonal, as the value of certain element $Q(i)[j, j]$ decreases, the steady-state accuracy of the corresponding estimate improves because the filter is more focused on rejecting the variations $t(i)$. Respectively, if $Q(i)[j, j]$ increases, the tracking ability improves at the cost of a lower measurement noise rejection. For ensuring that $Q(i)$ is in an appropriate order of magnitude, we compute its elements as

$$
Q(i)=\Upsilon \hat{P}(i) \Upsilon
$$

with

$$
\Upsilon^{2}=\left[\begin{array}{ccc}
v_{1} & & 0 \\
& \ddots & \\
0 & & v_{n_{j}}
\end{array}\right]
$$

and $v_{j} \in[0,1]$ chosen according to the desired performance. This leads to equation (40a) as

$$
\bar{P}(i)=\hat{P}(i-1)+\Upsilon \hat{P}(i-1) \Upsilon .
$$

Note that equation (48) excites more algorithm (40) when less knowledge of the states is available and, therefore, $\hat{P}(i)$ has a bigger value. This enhances the initialization of the algorithm.

Remark 5. The proposed algorithm implies computing equations (40) and (35) with $m(i)=y(i)^{\circ 2}$ every time that a new piece $i$ is manufactured in the MMP. At steady state, however, the gain matrix $L(i)$ is stationary. For reducing the computational burden, one can use the expressions

$$
L(i)=L(i-1), \hat{P}(i)=\hat{P}(i-1),
$$

instead of (40) whenever $|\hat{q}(i-1)-\hat{q}(i-2)| \leq \boldsymbol{\Delta}$ for some given difference $\Delta$.

Remark 6. Note that algorithm (40) provides a suboptimal Kalman gain due to the previous approximations and because $\Delta q(i)$ is non-zero when process variance changes occur. In any case, the previous design procedure presents the advantage of containing some numerical tuning parameters which can be used to set the trade-off between different estimation performance parameters.

\section{Estimator Properties}

The performance of the estimator depends on both the steady-state accuracy and on the delay in tracking the changes defined by $\Delta q(i)$.

1) Steady-state Behavior: Provided the unbiasedness of the estimator in fault-free scenarios (see Section III), the accuracy of the estimator (35) when $\Delta q(i)=0$ is given by the variance of the estimation errors, which we denote as $\phi_{j}$, i.e.,

$$
\phi_{j}=\operatorname{Var}\{\tilde{q}(i)[j] \mid \Delta q(i)=0\} .
$$

The steady-state covariance of the estimation errors due to noises, which we denote as $P$, is the solution of the following Riccati equation:

$$
P=\left(I_{n_{j}}-\bar{P} H^{T}\left(H \bar{P} H^{T}+T\right)^{-1} H\right) \bar{P},
$$

with $\bar{P}=P+\Upsilon P \Upsilon$, and where $T$ is the noise covariance at certain steady state. If we choose matrix $\Upsilon$ to have equal terms (i.e., $v_{j}=v, \forall j$ ), the covariance matrix of the steady-state error is given by

$$
P=\frac{v}{v+1}\left(H\left(\Sigma_{y} \circ \Sigma_{y}\right)^{-1} H^{T}\right)^{-1} .
$$

See Appendix $\mathrm{C}$ for the derivation of this expression. From (52), we deduce that the accuracy of the estimations improves as the design variables $v_{j}$ decrease.

2) Transient Behavior: The transient behaviour of the estimator can be characterized by the number of pieces whose data must be fed to the estimator in order to track certain change $\Delta q(i)[j]$. We define the settling time of the estimator, which we denote as $\eta_{90, j}$, as the number of pieces which are needed so that $\hat{q}[j]$ changes the $90 \%$ of the change experienced by $q[j]$ with $t(i)=0$. We can also describe the tracking ability of the estimator in terms of the cumulative squared error (CSE) experience by the $j$-th estimation due to process variance changes and which we define as

$$
\varphi_{j}=\sum_{i=1}^{\infty}\left\{\tilde{q}^{2}(i)[j] \mid t(i)=0\right\} .
$$

According to [33], the response of (35) when a unitary step change $\Delta q[j](i)$ occurs is like the response of a first-order system $g(i)$ defined as $g(i)=1-\left(1+v_{j}\right)^{-i}$ when $t(i)=0$. In this sense, the settling time and the CSE of the estimator due to unitary step changes are approximately given by

$$
\begin{aligned}
\eta_{90, j} & =\frac{-\log (0.1)}{\log \left(1+v_{j}\right)}, \\
\varphi_{j} & =\frac{\left(1+v_{j}\right)^{2}}{\left(1+v_{j}\right)^{2}-1},
\end{aligned}
$$

see [34]. From (54), we deduce that the tracking ability regarding the estimation of $q[j]$ improves when $v_{j}$ is increased.

Remark 7. Similarly to $\eta_{90, j}$ we can define $\eta_{\rho, j}$ as the number of pieces which are needed so that $\hat{q}[j]$ achieves the $\rho \%$ of the change experienced by $q[j]$ with $t(i)=0$ as

$$
\eta_{\rho, j}=\frac{-\log \left(\frac{100-\rho}{100}\right)}{\log \left(1+v_{j}\right)} .
$$




\section{Performance-based Observer Design}

As seen in Section IV-C, matrix $\Upsilon$ enhances the accomplishment of certain trade-off between the transient and the steadystate tracking performance. Relations (54a), (54b) and (51) can be used to choose the values $v_{j}\left(j=1, \ldots, n_{j}\right)$ so as to set certain trade-off regarding the performance of the estimator. For instance, in order to set certain settling time for the estimation of a unitary step change in $q[j]$, the variable $v_{j}$ must be fixed to

$$
v_{j}=0.1^{-1 / \eta_{90, j}}-1 \text {. }
$$

\section{Statistical Hypothesis Testing for Fault DIAGNOSIS}

In steady state (i.e., when no process variance changes occur), we denote the confidence interval offered by an estimate $\hat{q}(i)[j]$ for $q(i)[j]$ as

$$
\Omega_{j}(i)=\left[\hat{q}(i)[j]-h_{j}, \hat{q}(i)[j]+h_{j}\right],
$$

where $h_{j}$ depends on the confidence level of the interval, $\gamma_{j}=$ $1-\alpha_{j}$, and on the variance of the estimation $\hat{q}(i)[j]$. Note that, in steady state, the marginal variance of the estimation $\hat{q}[j]$ is $P[j, j]$, which can be obtained through equation (51).

Through Chebyshev's inequality [35], we have that

$$
\operatorname{Pr}\left\{q(i)[j] \notin \Omega_{j}(i)\right\} \leq P[j, j] / h_{j}^{2} .
$$

Then, if we set $h_{j}$ as

$$
h_{j}=\sqrt{P[j, j] / \alpha_{j}^{*}},
$$

we guarantee a bound $\alpha_{j} \leq \alpha_{j}^{*}$ for the confidence level of the interval $\Omega_{j}(i)$.

Relation (58) holds regardless of the probability distribution of $\hat{q}(i)[j]$. We know, however, that when $\Delta q(i)=0$, the estimation $\hat{q}(i)$ can be expressed as the infinite weighted sum

$$
\hat{q}(i)=-\sum_{\iota=1}^{i} \prod_{\kappa=\iota+1}^{i}\left(I_{n_{j}}-L(\kappa) H\right) L(\iota) t(\iota) .
$$

where the terms $-\prod_{\kappa=\iota+1}^{i}\left(I_{n_{j}}-L(\kappa) H\right) L(\iota)$ represent the weighting factors and $t(\iota)$ are independent and identically distributed zero-mean random variables with finite variance. One can demonstrate that the sequence of these weighting factors fulfills the conditions on [36] and then one can claim that $\hat{q}(i)$ approaches a normal distribution. Provided these results, we can set $h_{j}$ to fix the confidence level $\gamma_{j}$ to $1-\alpha_{j}^{*}$ as

$$
h_{j}=\Phi_{Z}^{-1}\left(1-\alpha_{j}^{*} / 2\right) \sqrt{P[j, j]},
$$

with $\Phi_{Z}^{-1}(\cdot)$ the inverse cumulative distribution function of a standard normal variable. This confidence level is tightener than the one obtained in (58) through Chebyshev's inequality.

Remark 8. Provided that $q(i) \geq 0$, we postprocess the value of the limits of $\Omega_{j}(i)$ so as to provide a refined confidence interval of $q(i)[j]$, which we denote as $\Omega_{j, p}(i)$ :

$$
\Omega_{j, p}(i)= \begin{cases}{\left[\hat{q}(i)[j]-h_{j}, \hat{q}(i)[j]+h_{j}\right]} & \text { if } \hat{q}(i)[j] \geq h_{j} \\ {\left[0, \hat{q}(i)[j]+h_{j}\right]} & \text { otherwise }\end{cases}
$$

Note that when we use the refined confidence interval $\Omega_{j, p}(i)$ in (62) and $h_{j}$ is defined through (59), we guarantee a bound $\alpha_{j} \leq \alpha_{j}^{*}$ which is looser than the bound which is guaranteed for $\Omega_{j}(i)$ in (57) (i.e., the difference between $\alpha_{j}$ and $\alpha_{j}^{*}$ is larger). Similarly, if $h_{j}$ is defined through (61) and we use the interval $\Omega_{j, p}(i)$ in (62), we just guarantee a bound $\alpha_{j} \leq \alpha_{j}^{*}$ instead of fixing its value to $\alpha_{j}=\alpha_{j}^{*}$ as happens with $\Omega_{j}(i)$ in (57). Thus, if we use $\Omega_{j, p}(i)$ in (62), the real confidence level is larger than the one that we have with $\Omega_{j}(i)$.

In order to determine whether a process variance of the MMP $q[j]$ has experienced a change $\Delta q[j]$ (i.e., a fault $j$ has appeared), we set the following statistical hypothesis test, where we use $i=0$ to refer to a piece for which the estimator has achieved the steady state and that has been manufactured with healthy conditions in the MMP:

$$
\left\{\begin{array}{ll}
\mathbf{H}_{\mathbf{0}}: & q(i)[j] \in \Omega_{j}(0) \\
\mathbf{H}_{\mathbf{1}}: & q(i)[j] \notin \Omega_{j}(0)
\end{array} .\right.
$$

Here, the null hypothesis stands for "No change of the $j$ th process variance" (i.e., "No fault $j$ ") and the alternative hypothesis stands for "Change of the $j$-th process variance" (i.e., "Fault $j "$ ).

\section{COMPARISON WITH BACTH-BASED ESTIMATORS}

As explained in Section II, the batch-based estimators such as the LSE or DSE are offline estimators which are conceived for periodically verify the state of the process. In the following, we compare the properties of these offline estimators with the characteristics of the proposed online approach.

- Accuracy of the estimations. In fault-free scenarios, the variance of the estimates provided by batch-based estimators is proportional to $1 / N$ (see [15] for details on the corresponding expressions). When using the proposed approach, the variance of the estimates is proportional to $v /(v+1)$ if every $v_{j}$ is fixed to a common value $v$ (see (52)).

- Time to track changes. The delay in tracking changes with the proposed online approach is approximately given by the settling time of the estimator which is defined by the number of pieces $\eta_{98, j}$ in (54a) and it is inversely proportional to $v_{j}$. For its part, offline batch-based methods just ensure the detection of a fault if a whole batch of faulty pieces is used in the computation of $S_{y}$ through (13). Then, these methods cannot ensure a tracking delay lower than $2 \mathrm{~N}$. Moreover, provided the non-zero variance of the estimates, the manufacturer might not state that a fault has occurred until certain number $\kappa$ of consecutive faulty estimations are available. In such a case, the number of faulty pieces which must be manufactured in order to state that a fault has occurred is $\eta_{98, j}+\kappa-1$ if the proposed approach is used and $\kappa N$ if offline estimators are used.

From these two considerations, we deduce that the existing trade-off between the accuracy of the estimations and their ability to track changes can be set through $\nu_{j}$ for the proposed approach (as explained in SectionIV-C) and through $N$ for batch-based methods. If $N$ is reduced (or if $\nu_{j}$ is increased), 
the tracking ability increases at the cost of lower estimation accuracies. For accuracies in the same order of magnitude (i.e., $\left.\frac{1}{N}=\frac{v}{v+1}\right)$, the time to track changes is $\kappa \frac{(v+1)}{v}$ for batchbased methods and $\kappa-1-\frac{\log (0.02)}{\log \left(1+v_{j}\right)}$ for the proposed approach. The tracking ability is thus in the same order of magnitude for both methods if $\kappa=1$ and it gets better for the proposed approach as $\kappa$ is increased.

Remark 9. Note that if big faults affect the system, it is not necessary that $\hat{q}[j]$ changes the $90 \%$ of the change experienced by $q[j]$ in order to detect a fault. If we use $\eta_{\rho, j}$ with $\rho<90$ instead of $\eta_{90, j}$ for the characterization of the tracking ability of the model-based observer, the time that we need in order to detect changes is exponentially reduced. Contrariwise, in no case this time is lower than $(\kappa-1) N$ for batch-based methods.

As stated in Section II, when it comes to estimators, other matters of fact are the computational burden, the volume of stored data and the design flexibility, which are now compared for both methods.

- Computational Burden.The algorithms of batch-based estimators (i.e., the LSE and the ASE) require $N$ vector product and summation operations, which are timeconsuming. As the accuracy of such methods improves when $N$ is increased, it happens that there exists a tradeoff between the accuracy of the estimations and the computational time of such methods. The computational burden of the proposed observer does not depend on the performance of the estimator because the variance of the estimation can be simply modified by changing the values in $\Upsilon$. Thus, the computational time is independent of the performance of the estimator.

- Data Storage. The sample size of the proposed approach only contains the data of the last manufactured piece. Thus, when certain piece $i$ is manufactured the stored data is $\hat{q}(i-1)$ and $y(i)$. For its part, the sample size of offline approaches contain the data of the last $N$ manufactured piece. Thus, when certain piece $i$ is manufactured the stored data is $y(i-N+1), y(i-N+1), \ldots, y(i-$ $1), y(i-2)$. Again, there exists a trade-off between the accuracy of the estimations and the amount of stored data which is not present in obser-based methods.

- Design Flexibility. The trade-off between the ability to track changes and the accuracy of the estimations is collectively set by $N$ for all the estimations $\hat{q}[j]$. Oppositely, this trade-off is individually set by $\nu_{j}$ for each the estimations $\hat{q}[j]$. Thus, the proposed approach enhances the use of different estimations performance requirements for each $q[j]$.

\section{CASE OF STUdY}

In this section, we set up different assembly processes in order to apply the strategies presented at the previous sections. Even if these processes are simple, the modeling and estimation framework is fairly general and it can be applied to more complex processes.
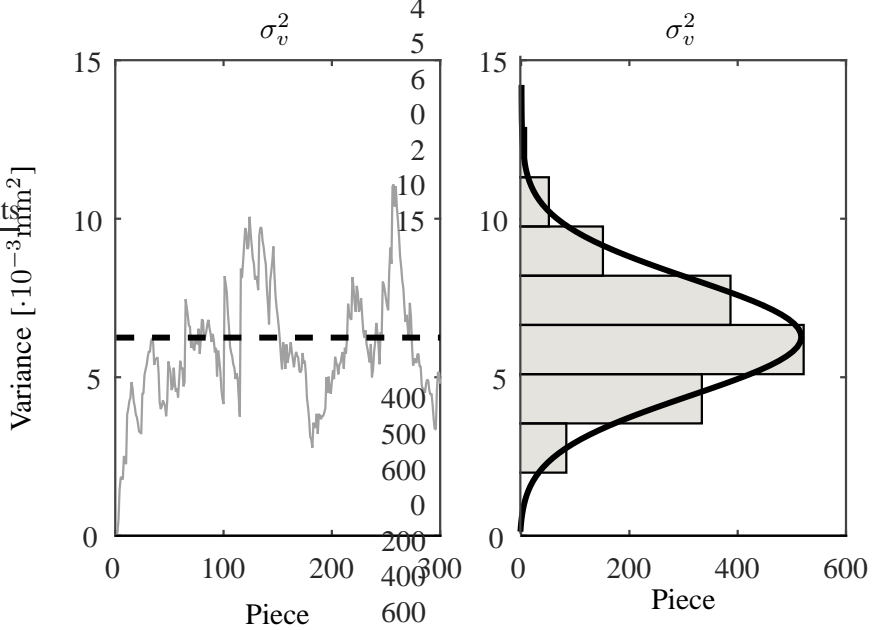

Figure 2. Stationary process variance estimation of $\sigma_{v}^{2}\left(v_{4}=0.05\right)$ in the single-stage assembly process. Real (- -), Estimation (Gray -), Fitted Gaussian distribution (Black -).

\section{A. Single-Stage Assembly Process}

First, we study a single-stage automotive body assembly described and modeled by Apley and Shi [16] and studied by Ding et al. [15]. In this single-stage case $(M=1)$, an optical coordinate measuring machine (OCMM) provides 9 measurements $\left(n_{y}=9\right)$. The pieces at this stage are fixed by a 4-way locator, $P_{1}$, which produces positioning variability in two directions (i.e., $\delta P_{1}^{x}$ and $\delta P_{1}^{z}$ ) and by a 2-way locator, $P_{2}$, which only produces positioning variability in one direction (i.e., $\delta P_{2}^{z}$ ); thus, $u_{k}=\left[\delta P_{1, k}^{x} \delta P_{1, k}^{z} \delta P_{2, k}^{z}\right]^{T}$ and $n_{u}=3$. The replicated matrix $\Gamma$ is

$$
\Gamma=\left[\begin{array}{ccc}
0.093 & 0.577 & -0.120 \\
0 & 0 & 0 \\
-0.093 & 0 & 0.843 \\
0.093 & 0.577 & -0.120 \\
0 & 0 & 0 \\
0.647 & 0 & -0.120 \\
-0.370 & 0.577 & 0.482 \\
0 & 0 & 0 \\
0.647 & 0 & -0.120
\end{array}\right] .
$$

The sensor accuracy is $(6 \sigma)_{\text {sensor }}=0.1 \mathrm{~mm}$ and the tolerance of the pinholes is $0.2 \mathrm{~mm}$. If the tolerance is approximated by the six-sigma value; then, $(6 \sigma)_{\text {locator }}=0.2 \mathrm{~mm}$.

First, we simulate an stationary process of 300 pieces with $q=\left[\begin{array}{llll}1.1 & 2.5 & 04.4 & 0.6\end{array}\right]^{T} \cdot 10^{-3} \mathrm{~mm}^{2}$ (values taken from [15]). The left-hand side of Fig.2 shows the estimation results for $q[4]$ (i.e., $\hat{q}_{p}[4]$ ) when applying an observer with $\Upsilon^{2}=0.05 I_{4}$ in (46). On the right-hand side of Fig.2, we look at the shape of the probability distribution of $\hat{q}_{p}[4]$ when running 50 simulation trials and we compare it with a fitted normal density proving a high goodness of fit of the Gaussian distribution. Similar results apply to the other variance estimations $\hat{q}_{p}[j]$ with $j=\{1,2,3\}$, which we do not include due to space constraints. The variance of the estimations provided by different observers are shown in Table I. 
Table I

VARIANCE OF THE STEADY-STATE ESTIMATIONS $\left[\cdot 10^{-6} \mathrm{~mm}^{4}\right]$ FOR DIFFERENT OBSERVERS WITH $\Upsilon^{2}=v I_{4}$

\begin{tabular}{ccccc}
\hline$v$ & $\operatorname{Var}\left\{\hat{q}_{p}[1]\right\}$ & $\operatorname{Var}\left\{\hat{q}_{p}[2]\right\}$ & $\operatorname{Var}\left\{\hat{q}_{p}[3]\right\}$ & $\operatorname{Var}\left\{\hat{q}_{p}[4]\right\}$ \\
\hline 0.0010 & 0.0217 & 0.0103 & 0.0166 & 0.0001 \\
0.0152 & 0.1362 & 0.1784 & 0.3443 & 0.0029 \\
0.0294 & 0.2322 & 0.3551 & 0.6693 & 0.0051 \\
0.0436 & 0.3182 & 0.5384 & 1.0273 & 0.0071 \\
0.0579 & 0.4010 & 0.7288 & 1.4120 & 0.0090 \\
0.0721 & 0.4880 & 0.9272 & 1.8032 & 0.0109 \\
0.0863 & 0.5773 & 1.1415 & 2.2138 & 0.0128 \\
0.1005 & 0.6698 & 1.3773 & 2.6695 & 0.0147 \\
0.1147 & 0.7640 & 1.6326 & 3.1927 & 0.0167 \\
0.1289 & 0.8599 & 1.9116 & 3.8059 & 0.0186 \\
0.1431 & 0.9600 & 2.2181 & 4.5634 & 0.0205 \\
0.1574 & 1.0698 & 2.5425 & 5.5490 & 0.0224 \\
0.1716 & 1.1817 & 2.9035 & 6.7644 & 0.0243 \\
0.1858 & 1.2843 & 3.3121 & 8.1135 & 0.0261 \\
0.2000 & 1.3797 & 3.7668 & 9.6098 & 0.0280 \\
\hline
\end{tabular}

In order to compare the proposed online estimators with the process variance estimators based on the sampling variance of a batch of $N$ pieces, we apply these estimators online by inferring a time-varying population variance every time that a new piece $i$ is manufactured from $S_{y}(i)$ computed through (14) (i.e., every time that a new piece $i$ is entirely measured, the estimation is made with the data provided by this piece and by the previous $N-1$ completed pieces). In Fig. 3, we compare the relative computational time which is needed to perform the estimation of the previous process for different estimators and different values of the corresponding tuning parameters that lead to different variances of the estimations. As explained in Section VI, Fig. 3 shows that the computational burden of the proposed observer (KF) does not depend on the performance of the estimator because the variance of the estimation can be simply modified by changing the values in $\Upsilon$. Oppositely, the algorithms of batchbased estimators (i.e., the LSE and the ASE) require more operations as the estimation accuracy is improved and, thus, the computational time increases with the accuracy. From this figure, we also deduce that the estimator (35)-(40) is more computationally costly than the LSE and the ASE for estimations of lower accuracies. However, our estimator is more efficient and becomes more computationally appealing as the desired accuracy of the estimations is increased. Moreover, if we use relaxation (49) (steady-state KF), the computational burden is dramatically reduced for all accuracies.

Fig.4 shows the results provided by three observers with different performance w.r.t. the estimation of $q[4]$ when the process of 300 pieces is affected by an abrupt fault which modifies the sensors accuracy from $0.6 \cdot 10^{-3} \mathrm{~mm}^{2}$ to 0.95 . $10^{-3} \mathrm{~mm}^{2}$ at the piece 150 . The observers have been designed with requirements over the CSE due to unitary step changes $\Delta q(4): \varphi_{4}=5$ (giving $v_{4}=0.1$ ), $\varphi_{4}=10$ (giving $v_{4}=$ 0.05 ) and $\varphi_{4}=15$ (giving $v_{4}=0.01$ ). We prove that, as explained in Section IV, a smaller CSE (and higher tuning parameter in $\Upsilon$ ) results in a better tracking ability at the cost of a lower performance w.r.t. the variance of the process variance estimations. The designer should tune this parameter according to the criticality of the delays in tracking changes.

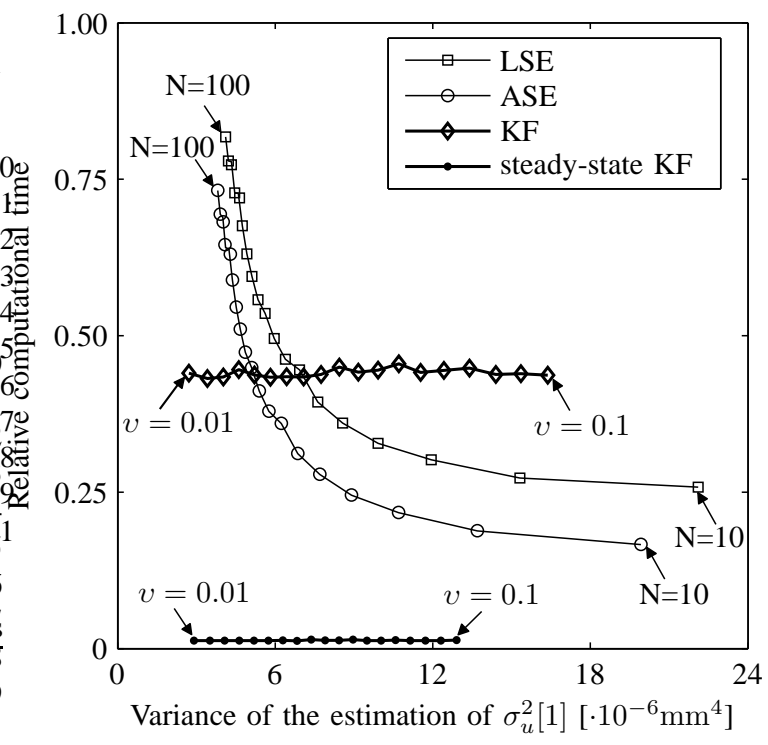

Figure 3. Comparison of the computational time which is needed for different estimation accuracies regarding $\sigma_{u}^{2}[1]$ at steady state with different estimation strategies.

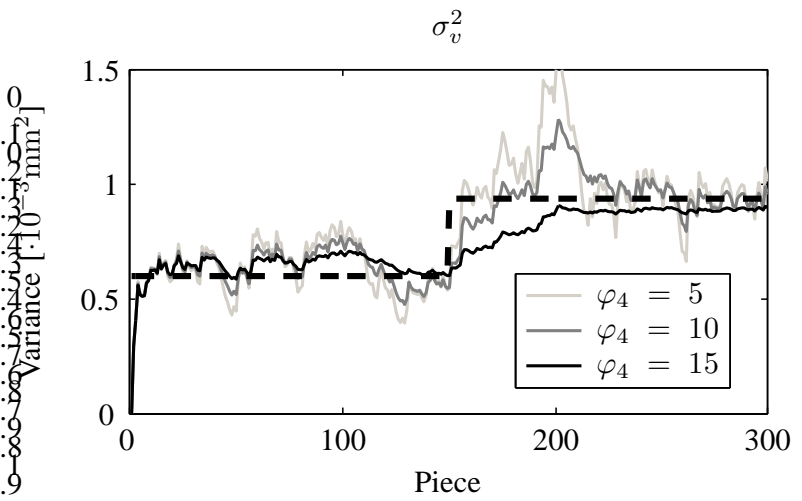

Figure 4. Comparison of the performance of different observers used in the single-stage assembly process for the estimation of $\sigma_{v}^{2}$, which is affected by an abrupt fault. Real (- -), Estimations (-).

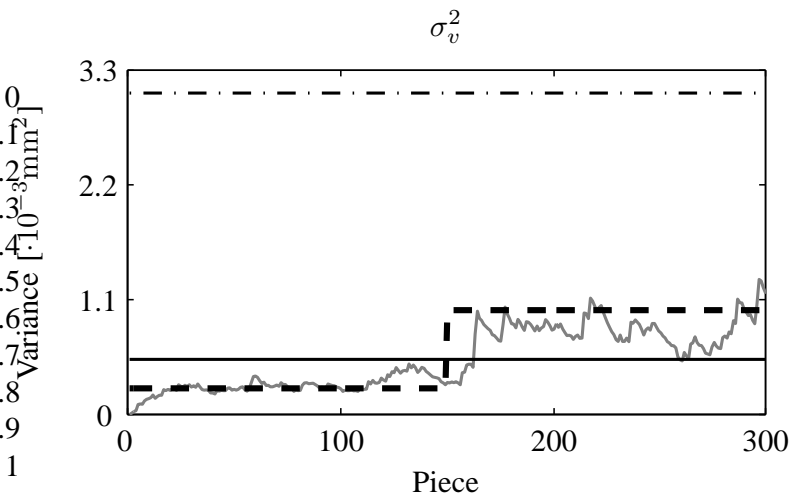

Figure 5. Comparison of statistical testing methods with different confidence interval limits $\left(\gamma_{j}=99 \%\right)$ for the estimation of $\sigma_{v}^{2}\left(v_{4}=0.05\right)$ in the singlestage assembly process. Real (- -), Estimation (Gray -), Gaussian-based limit (Black -), Chebyshev-based limit (--).

In Fig.5, we include the estimation results of $q[4]$ provided by the model-based observer designed with $\Upsilon^{2}=0.05 I_{4}$ together with the limits of $\Omega_{4, p}(0)$ for both the computation of $h_{4}$ through (59) and through (61) $\left(\gamma_{4}=99 \%\right)$. When no taking account of the Gaussian behaviour of the estimations 

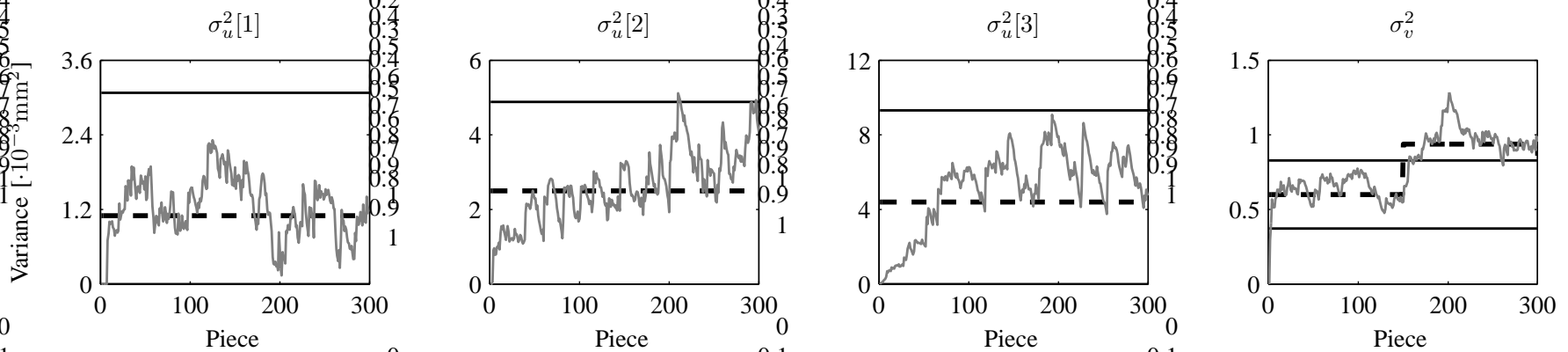

Figure 6. Process variance estimation with a model-based observer $\left(\Upsilon^{2}=0.05 I_{4}\right)$ in the single-stage assembly process affected by an abrupt fault in $\sigma_{v}^{2}$. Real (- -), Estimation (Gray -) Gaussian-based limit with $\gamma_{j}=99 \%$ (Black -).
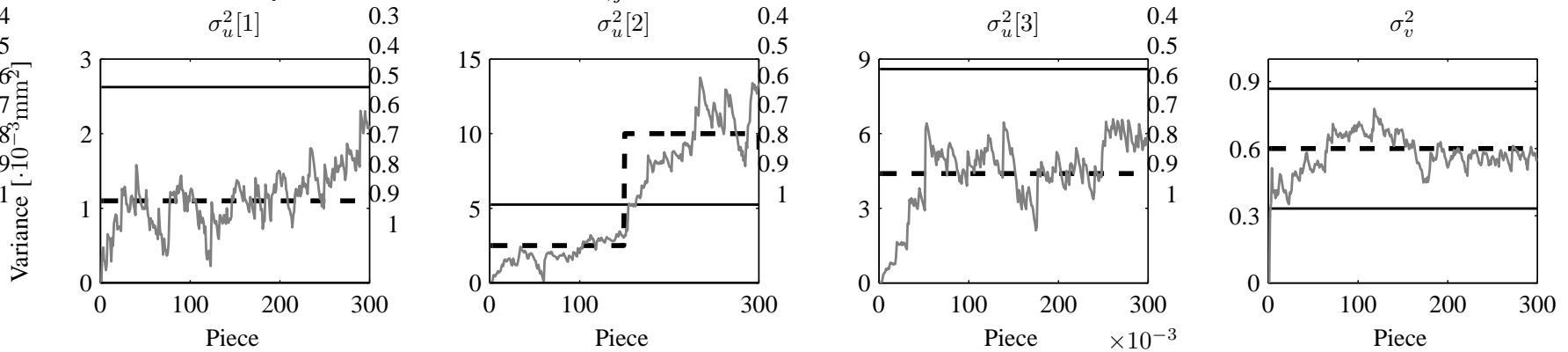

Figure 7. Process variance estimation with a model-based observer $\left(\Upsilon^{2}=0.05 I_{4}\right)$ in the single-stage assembly process affected by an abrupt fault in $\sigma_{u}^{2}[2]$. Real (- -), Estimation (Gray -) Gaussian-based limit with $\gamma_{j}=99 \%$ (Black -).

and the limits are computed by means of the Chebyshev's inequality, the confidence interval $\Omega_{4, p}(0)$ is too big and only big variance changes can be diagnosed. On the contrary, the limits of $\Omega_{4, p}(0)$ when computed through (61) are tight and smaller faults (as the one being simulated) can be diagnosed.

Thus, in Fig.6 we include all the estimations provided by the estimator $\left(\Upsilon^{2}=0.05 I_{4}\right)$ for this process with the corresponding the Gaussian-based thresholds of $\Omega_{j, p}(0)\left(\gamma_{j}=99 \%\right.$ with $j=\{1,2,3,4\}$ ). We prove that, although the order of magnitude of the variables involved in $q(i)$ is different, the model-based observer provides appropriate results. Note that the observer depends on $T(i)$, which is a function of $q(i)$ (see (40)-(44)). Thus, when certain component of $q(i)$ increases its value, so does the variance of the estimations. Fig.7 shows, for its part, the simulation results (with $\Upsilon^{2}=0.05 I_{4}$ and $\gamma_{4}=99 \%$ ) for the process in which we suppose that $\delta P_{1, k}^{z}$ is affected by an abrupt fault, which doubles its standard deviation from $0.05 \mathrm{~mm}$ to $0.1 \mathrm{~mm}$ at the piece 150 . Then, we have that $q(i)$ in $\mathrm{mm}^{2}$ is now given by

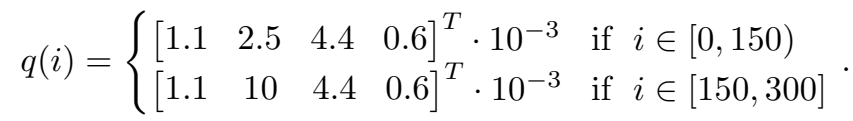

As shown in the corresponding figure, this fault is detected in 52 pieces. If for the simulated fault $\left(\Delta q[3]=7.5 \cdot 10^{-3} \mathrm{~mm}^{2}\right)$ this number of corrupted pieces is critical, the designer may decide to build an estimator with higher tuning parameters in $\Upsilon^{2}$ at the cost of poorer estimations in terms of accuracy.

For the simulations in Fig. 6 and Fig.7 we have assumed that no a priori knowledge of the variances $\sigma_{u}^{2}[j]$ and $\sigma_{v}^{2}$ is available. Then, the estimator should be initialized at $\hat{q}(0)[j]=0$ and the estimations increase progressively in value as new measurements are processed. Moreover, some simulation results in these figures may look as a fault appeared when it does not (e.g., the variation experienced by $\sigma_{u}^{2}[2]$ in Fig.6). The reader should notice that these variations are within the confidence intervals designed for a $99 \%$ confidence level. If just thinner variations are allowed, the designer should decrease the value of the variables $\nu_{j}$ so that the accuracy of the estimates improves; contrariwise, if these variations should fade faster, the designer should increase the value of the variables $\nu_{j}$ so that the tracking ability of the estimator improves. In Fig. 8 we show the estimation of $\sigma_{u}^{2}[1]$ for a fault-free scenario. If we use an observer with $\Upsilon^{2}=0.1 I_{4}$, the latest simulation results may look as if an abrupt fault appeared but, in any case, the estimation is within the confidence interval for $\gamma_{1}=99 \%\left(\Omega_{j, p}(i)=[0,3.236] \cdot 10^{-3}\right)$ and no fault is thus detected. If this temporary bias is prohibitive in terms of accuracy, one can use other observers as the ones in the second part of Fig.8, which are designed with $\Upsilon^{2}=0.01 I_{4}\left(\Omega_{j, p}(i)=[0.315,1.885] \cdot 10^{-3}\right.$ for $\left.\gamma_{1}=99 \%\right)$ and $\Upsilon^{2}=0.001 I_{4}\left(\Omega_{j, p}(i)=[0.493,1.707] \cdot 10^{-3}\right.$ for $\left.\gamma_{1}=99 \%\right)$.

\section{B. Multistage Assembly Process}

Second, we study the two-stage process $(\mathrm{M}=2)$ provided in [15] which was derived from a segment of the simplied automotive body assembly process [18]. This simplified assembly process has been widely used in the literature for analysing diagnosability issues [18], [37], estimation of variance components of variation sources [15] and optimal sensor distribution [38], [39]. In this example, depicted in Fig.9, three workpieces are welded together at Stage I. In this stage, there are 9 fixturing variation sources $\left(n_{u}=9\right)$. Once welding operations are completed, the entire assembly is transferred 


$$
\Gamma=\left[\begin{array}{ccccccccc}
0 & 0 & 0 & 0.1215 & -0.3846 & 0 & 0 & 0 & 0.2632 \\
0 & 0 & 0 & 0.0221 & -0.0699 & 0 & 0 & 0 & 0.0478 \\
0 & 0 & 0 & 0.1215 & -0.3846 & 0 & 0 & 0 & 0.2632 \\
0 & 0 & 0 & -0.1817 & 0.5944 & 0 & 0 & 0 & -0.4067 \\
0 & 0 & 0 & -0.0773 & 0.2448 & 0 & 0 & 0 & -0.1675 \\
0 & 0 & 0 & -0.3379 & 1.0699 & 0 & 0 & 0 & -0.7321 \\
0 & 0 & 0 & 0.1656 & -0.5245 & 0 & 0 & 0 & 0.3589 \\
0 & 0 & 0 & -0.3379 & 1.0699 & 0 & 0 & 0 & -0.7321 \\
0 & 0 & 0 & 0 & 0 & 0 & 0 & 0 & 0 \\
0 & 0 & 0 & -0.2054 & 0.6503 & 0 & 0 & 0 & -0.445 \\
-1 & 1 & 0 & -0.3110 & 0 & 0.4 & -0.4 & 0 & 0.311 \\
0 & 0 & 0 & 0.0574 & 0 & -0.24 & 1.24 & 0 & -1.0574 \\
-1 & 1 & 0 & -0.2153 & 0 & 0 & 0 & 0 & 0.2153 \\
0 & 0 & 0 & -0.2392 & 0 & 1 & 0 & 0 & -0.7608 \\
-1 & 0 & 1 & -0.0957 & 0 & 0 & 0 & 0.4 & -0.3043 \\
0 & 0 & 0 & 0.0574 & 0 & 0 & 0 & -0.24 & 0.1826 \\
-1 & 0 & 1 & 0 & 0 & 0 & 0 & 0 & 0 \\
0 & 0 & 0 & -0.2392 & 0 & 0 & 0 & 1 & -0.7608
\end{array}\right] .
$$
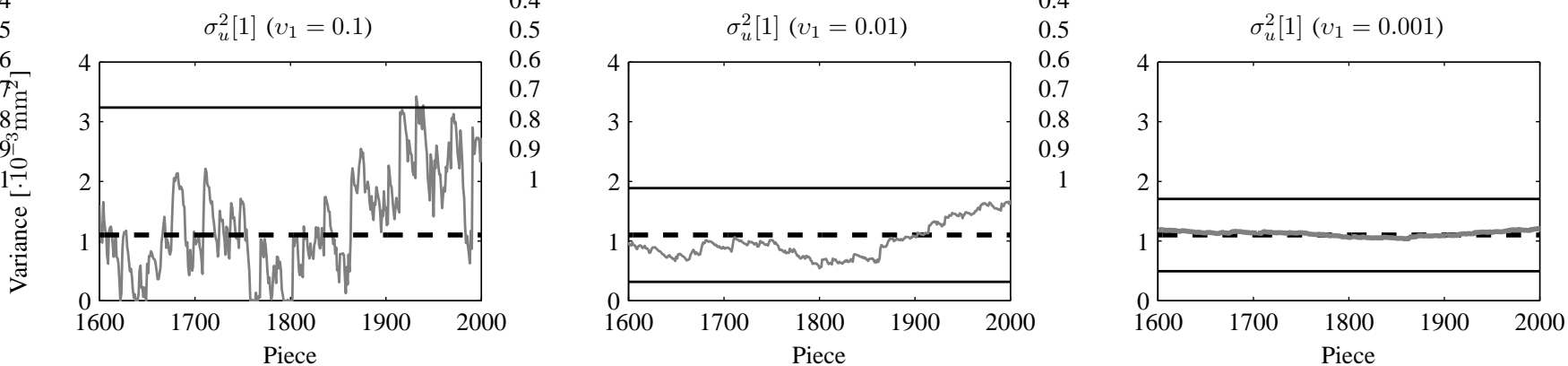

Figure 8. Comparison of the performance of different observers used in the single-stage assembly process for the estimation of $\sigma_{u}^{2}[1]$ in fault-free scenarios. Real (- -), Estimation (Gray -) Gaussian-based limit with $\gamma_{1}=99 \%$ (Black -).

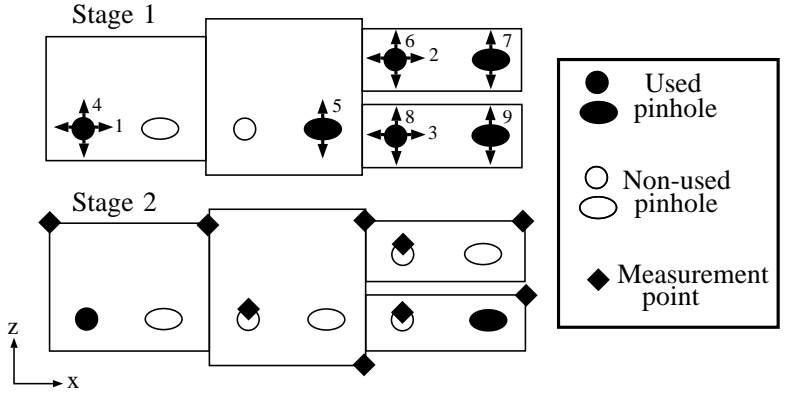

Figure 9. Scheme of the two-stage assembly process, adapted from [15], [18].

to a dedicated in-process OCMM stage (Stage II) for inspection. High-precision laser-optic coordinate sensors are used to measure two directional coordinates at each measurement point and $n_{y}=18$. Matrix $\Gamma$ is given in (64).

The output matrix $H$ which results from $\Gamma$ does not verify condition (43); then, we redefine vector $q(i)$ so that $n_{j}=$ $\operatorname{rank}\{H\}$ as

$$
q(i)[j]=\left\{\begin{array}{ll}
\sigma_{u}^{2}[1]+\sigma_{u}^{2}[2] & \text { if } j=1 \\
\sigma_{u}^{2}[1]+\sigma_{u}^{2}[3] & \text { if } j=2 \\
\sigma_{u}^{2}[j+1] & \text { if } j \in\{3,4,5,6,7,8\} \\
\sigma_{v}^{2} & \text { if } j=9
\end{array} .\right.
$$

and we modify matrix $H$ accordingly. This means that in the case of the three first fixturing variation sources we do not identify the process variations individually, but a combination of them.

First, we simulate a process of 2250 pieces with the values

$$
\sigma^{2}(i)[j]=\left\{\begin{array}{ll}
4.5 \cdot 10^{-3} & \text { if } j=1 \\
0.3 \cdot 10^{-3} & \text { if } j \in\{2,3\} \\
0.5 \cdot 10^{-3} & \text { if } j \in\{4,5,8,9\} \\
1.5 \cdot 10^{-3} & \text { if } j=6 \\
2.5 \cdot 10^{-3} & \text { if } j=7 \\
0.0111 \cdot 10^{-3} & \text { if } j=10
\end{array} .\right.
$$

(values in $\mathrm{mm}^{2}$ taken from [15]). We assume that an abrupt change occurs modifying the variance $\sigma_{u}^{2}[6]$ from $1.5 \cdot 10^{-3}$ $\mathrm{mm}^{2}$ to $3.4 \cdot 10^{-3} \mathrm{~mm}^{2}$ (i.e., doubled standard deviation of the 6-th variation source) at piece 1000. Fig.10 shows the estimation results regarding $\sigma_{u}^{2}[j]$ when applying an observer with $\Upsilon^{2}=0.002 I_{9}$ in (46) and $\gamma_{j}=99 \%$. Again, we prove that, although the order of magnitude of the variables involved in $q(i)$ is highly different, the model-based observer provides appropriate results.

Now, we simulate a process of 1200 pieces in which standard deviation of the sensors is modified from $0.0033 \mathrm{~mm}$ to $0.0100 \mathrm{~mm}$ at piece 250 . The first simulation depicted in 

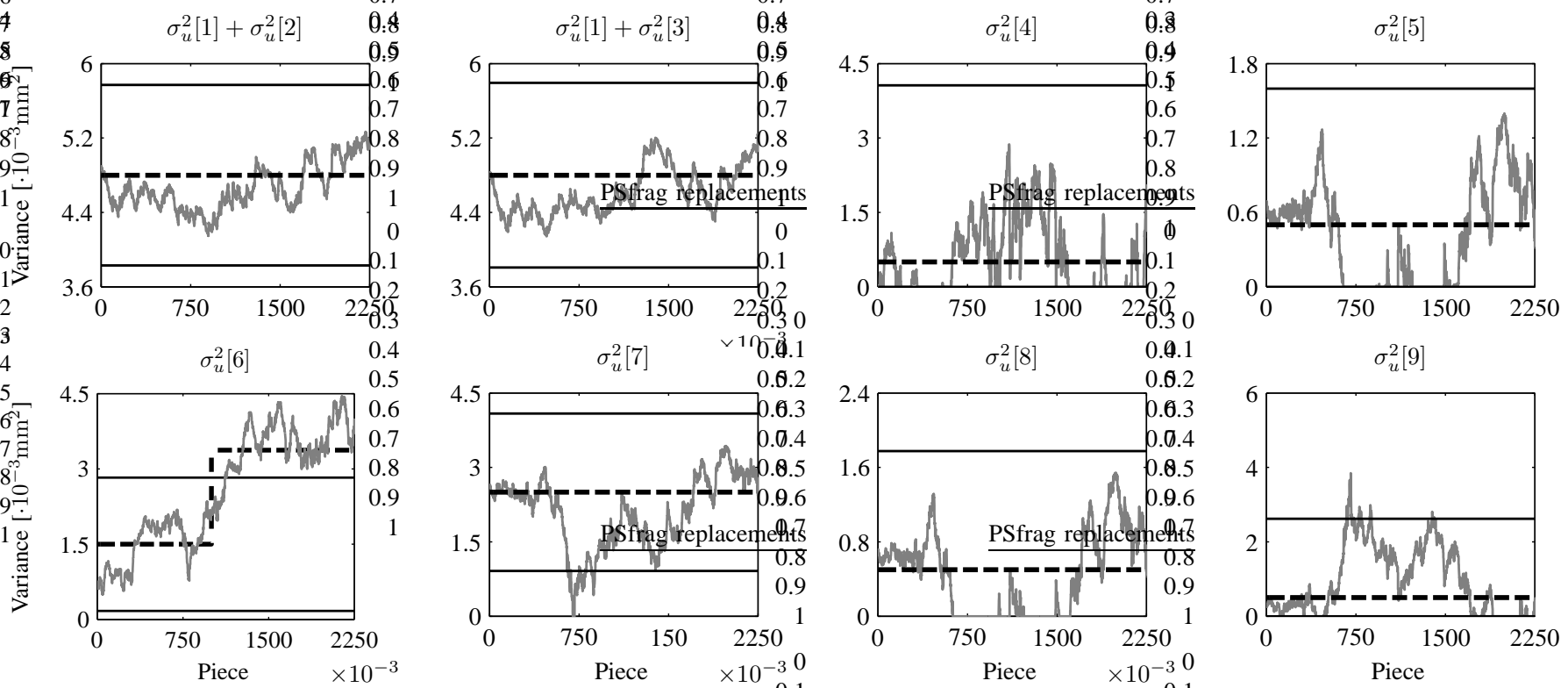

Figure 10. Process variance estimation with a model-based observer $\left(\Upsilon^{2}=0.002 I_{9}\right)$ in the multistage assembly process affected by an abrupt fault in $\sigma_{u}^{2}[6]$ Real (- -), Estimation (Gray -) Gaussian-based limit with $\gamma_{j}=99 \%$ (Black -).

Fig.11 shows the estimation results for this process when using an estimator designed with $\nu_{9}=0.002$ and a Gaussian-based threshold with $\gamma_{9}=99 \%$. The second part of this figure shows that the proposed algorithm (with $\nu_{9}=0.002$ and $\gamma_{9}=99 \%$ ) does also track drift faults. In this case, $\sigma_{v}=0.0033 \mathrm{~mm}$ before the drift fault and $\sigma_{v}=0.0073 \mathrm{~mm} 1000$ pieces after the fault appears. The drift fault modifies the sensor process variance linearly. The other results compare the performance of estimators with different requirements over the estimation of $q[9]: v_{1}=0.01$, and $v_{1}=0.001$. Again, we see the tradeoffs detailed in Section IV. Note that the order of magnitude of the tuning parameter (i.e., $v_{j}$ ) used in the estimators in Fig.11 is smaller than the order of magnitude of the tuning parameter in the estimators in Fig.4 due to the differences in the values involved in the MMP.

\section{CONCLUSION}

In this work, we have addressed the non-stationary process variance estimation problem with a recursive algorithm that is updated with the information available from every new manufactured piece. This strategy relaxes the computational burden and the data storage required by other algorithms that use a large sample size for each computation. Furthermore, our approach has a multivariate parameter that tunes the performance in the existing trade-off between the adaptation to process variance changes and the accuracy in stationary periods. We have shown two possible computations of the confidence interval, one based on the Chevyshev's inequality and another which takes account of the Gaussian behavior which is approached by the estimation errors. We have shown in two different examples how to tune the algorithm in order to obtain a desired performance. Future work will include the application of the proposed approach to factory collected and the extension of the approach to obtain not only process
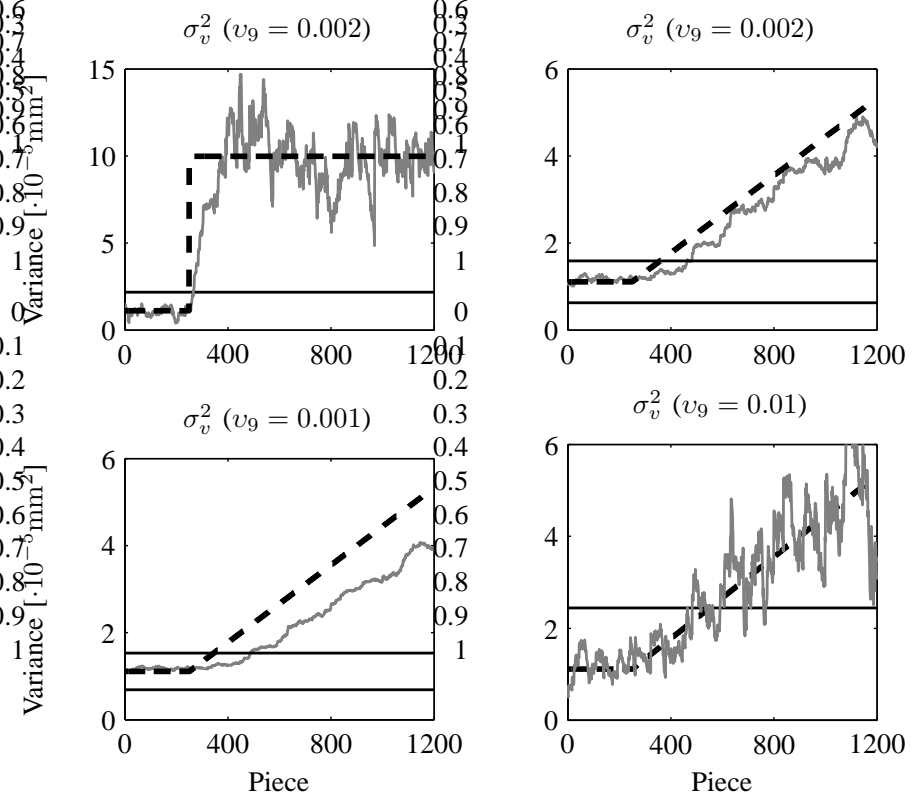

Figure 11. Comparison of the performance of different observers used in the multistage assembly process for the estimation of $\sigma_{v}^{2}$, which is affected by an abrupt fault or a drift fault. Real (- -), Estimation (Gray - ) Gaussian-based limit with $\gamma_{j}=99 \%$ (Black -).

variance estimations but also estimations of the mean of the variation sources.

\section{APPENDIX}

\section{A. Derivation of the expected value of $m(i)$}

Let us first introduce the following lemma.

Lemma 2. ([40]) Let $A \in \mathbb{R}^{n_{a} \times n}, X \in \mathbb{R}^{n \times n}$ and $B \in$ $\mathbb{R}^{n_{b} \times n}$ be some matrices. We have that

$$
\operatorname{vec}\left(A X B^{T}\right)=B \otimes A \operatorname{vec}(X) .
$$


If matrix $X$ is diagonal, we have that

$$
\operatorname{diag}\left(A X A^{T}\right)=A^{\circ 2} \operatorname{diag}(X) .
$$

Provided (25), we have that

$$
\mathbf{E}\left\{y(i) y(i)^{T}\right\}=\mathbf{E}\left\{\Psi(i) \zeta(i) \zeta(i)^{T} \Psi(i)^{T}\right\} .
$$

Applying (65) to (67), we get

$$
\mathbf{E}\left\{y(i) y(i)^{T}\right\}=\operatorname{vec}^{-1}\left(\Psi(i)^{\otimes^{2}} \operatorname{vec}\left(\mathbf{E}\left\{\zeta(i) \zeta(i)^{T}\right\}\right)\right),
$$

where we have taken into account that $\mathbf{E}\{\Psi(i)\}=\Psi(i)$. Provided (20), this relation is simplified as

$$
\mathbf{E}\left\{y(i) y(i)^{T}\right\}=\Psi(i) \Psi(i)^{T} .
$$

Taking into account that matrices $D(i) D(i)^{T}$ and $R(i) R(i)^{T}$ are diagonal, we apply (66) to (69) and we get that the diagonal components of $\mathbf{E}\left\{y(i) y(i)^{T}\right\}$ are given by

$$
\operatorname{diag}\left(\mathbf{E}\left\{y(i) y(i)^{T}\right\}\right)=\Gamma^{\circ 2} \operatorname{diag}\left(\boldsymbol{\Sigma}_{u}(i)\right)+\mathbf{1}_{n_{y}} \sigma_{v}^{2}(i),
$$

which can be rewritten as

$$
\mathbf{E}\{m(i)\}=\left[\begin{array}{ll}
\Gamma^{\circ 2} & \mathbf{1}_{n_{y}}
\end{array}\right] \operatorname{diag}\left(\bigoplus_{j} \sigma^{2}(i)[j]\right) .
$$

\section{B. Kalman filtering}

Let us consider a linear system of the form

$$
x(i+1)=A x(i)+w(i), \quad y(i)=C x(i)+v(i),
$$

with $x \in \mathbb{R}^{n_{x}}$ the state vector, $y \in \mathbb{R}^{n_{y}}$ the output vector, $A \in \mathbb{R}^{n_{x} \times n_{x}}$ the state matrix and $C \in \mathbb{R}^{n_{y} \times n_{x}}$ the output matrix. Vector $w(i) \in \mathbb{R}^{n_{w}}$ contains the process noise and $v(i) \in \mathbb{R}^{n_{w}}$ contains the measurement noise. We assume that $w(i)$ and $v(i)$ are independent, zero-mean Gaussian noises of time-varying covariances $W(i)=\mathbf{E}\left\{w(i) w(i)^{T}\right\}$ and $V(i)=$ $\mathbf{E}\left\{v(i) v(i)^{T}\right\}$. A model-based observer for (72) is

$$
\hat{x}(i)=A \hat{x}(i-1)+L(i)(y(i)-C A \hat{x}(i-1)),
$$

with $\hat{x}(i)$ the estimated state, see [41]. Then, the dynamics of the estimation error $\tilde{x}(i)=x(i)-\hat{x}(i)$ is

$$
\tilde{x}(i)=\left(I_{n_{x}}-L(i) C\right)(A \tilde{x}(i-1)+w(i-1))-L(i) v(i) .
$$

The optimal Kalman gain $L(i)$ for (73) is given by

$$
\begin{aligned}
\bar{P}(i) & =A \hat{P}(i-1) A^{T}+Q(i-1), \\
L(i) & =\bar{P}(i) C^{T}\left(C \bar{P}(i) C^{T}+V(i)\right)^{-1}, \\
\hat{P}(i) & =\left(I_{n_{x}}-L(i) C\right) \bar{P}(i),
\end{aligned}
$$

see [31], [32]. Here, $\bar{P}(i)$ and $\hat{P}(i)$ represent, respectively, the predicted and the estimation error covariance matrix.

\section{Derivation of the Statistical Properties of the Estimator}

Let us first remember the Woodbury matrix identity [42]:

$$
(D+E F G)^{-1}=D^{-1}-D^{-1} E\left(F^{-1}+G D^{-1} E\right)^{-1} G D^{-1} \text {. }
$$

Now, assuming $v_{j}=v$, for all $j$, we rewrite (51) as

$$
P=\left(I-\nu P H^{T}\left(H \nu P H^{T}+T\right)^{-1} H\right) \nu P
$$

with $\nu=1+v$. Premultiplying and postmultiplying (77) by $(\nu P)^{-1}$, and applying (76) to the term $\left(H \nu P H^{T}+T\right)^{-1}$ (with $D=T, E=H, F=\nu P$ and $G=H^{T}$ ) it leads to

$$
\left(\nu^{-1}-\nu^{-2}\right) P^{-1}=\Xi-\Xi\left(\nu^{-1} P^{-1}+\Xi\right)^{-1} \Xi
$$

with $\Xi=H^{T} T^{-1} H$. Now, applying again (76) to the term $\left(\nu^{-1} P^{-1}+\Xi\right)^{-1}$ (with $D=\Xi, E=I, F=\nu^{-1} P^{-1}$ and $G=I)$ we obtain that

$$
\left(\nu^{-1}-\nu^{-2}\right) P^{-1}=\left(\nu P+\Xi^{-1}\right)^{-1} .
$$

Inverting both sides of the equality and substituting $\Xi=$ $H^{T} T^{-1} H$, we finally have that

$$
P=\frac{\nu-1}{\nu}\left(H^{T} T^{-1} H\right)^{-1} \text {. }
$$

For its part, at steady state, $T$ is given by $T=\left(\Psi \Psi^{T}\right)^{\circ 2}$ and

$$
\Psi \Psi^{T}=[\Gamma D R]\left[\begin{array}{ll}
\Gamma D & R
\end{array}\right]^{T}=\Gamma D D^{T} \Gamma+R R^{T} .
$$

From the definition of matrices $D$ and $R$ and using relation (5), we have that, at steady state,

$$
\Psi \Psi^{T}=\Gamma \Sigma_{u}^{T} \Gamma+\Sigma_{v}=\Sigma_{y} .
$$

Then, matrix $T$ can be expressed as

$$
T=\left(\Psi \Psi^{T}\right)^{\circ 2}=\Sigma_{y} \circ \Sigma_{y} .
$$

Finally, using (83) in (80) and substituting $\nu=1+v$, we get (52).

\section{REFERENCES}

[1] J. Shi and S. Zhou. Quality control and improvement for multistage systems: A survey. IIE Transactions, 41(9):744-753, 2009.

[2] J. Li, J. Jin, and J. Shi. Causation-based $T^{2}$ decomposition for multivariate process monitoring and diagnosis. Journal of Quality Technology, 40(1):46-58, 2008.

[3] S. Verron, J. Li, and T. Tiplica. Fault detection and isolation of faults in a multivariate process with bayesian network. Journal of Process Control, 20(8):902-911, 2010.

[4] R. Mantripragada and D.E. Whitney. Modeling and controlling variation propagation in mechanical assemblies using state transition models. IEEE Transactions on Robotics and Automation, 15(1):124-140, 1999.

[5] J. Jin and J. Shi. State space modeling of sheet metal assembly for dimensional control. Journal of Manufacturing Science and Engineering, 121(4):756-762, 1999.

[6] S. Zhou, Q. Huang, and J. Shi. State space modeling of dimensional variation propagation in multistage machining process using differential motion vectors. IEEE Transactions on Robotics and Automation, 19(2):296-309, 2003.

[7] J. Liu, J. Jin, and J. Shi. State space modeling for 3-D variation propagation in rigid-body multistage assembly processes. IEEE Transactions on Automation Science and Engineering, 7(2):274-290, 2010.

[8] J.V. Abellan-Nebot, J. Liu, F. Romero-Subirón, and J. Shi. State space modeling of variation propagation in multistation machining processes considering machining-induced variations. Journal of Manufacturing Science and Engineering, 134(2):021002, 2012. 
[9] J.V. Abellán-Nebot, I. Peñarrocha, E. Sales-Setién, and J. Liu. Optimal inspection/actuator placement for robust dimensional compensation in multistage manufacturing processes. In Computational Methods and Production Engineering, pages 31-50. Elsevier, 2017.

[10] F. Yang, S. Jin, and Z. Li. A comprehensive study of linear variation propagation modeling methods for multistage machining processes. The International Journal of Advanced Manufacturing Technology, 90(58):2139-2151, 2017.

[11] F. Yang and Z. Jin, S.and Li. A modification of dmvs based state space model of variation propagation for multistage machining processes. Assembly Automation, 37(4):381-390, 2017.

[12] C. Zou and F. Tsung. Directional MEWMA schemes for multistage process monitoring and diagnosis. Journal of Quality Technology, 40(4):407-427, 2008

[13] S. Zhou, Y. Chen, and J. Shi. Statistical estimation and testing for variation root-cause identification of multistage manufacturing processes. IEEE Transactions on Automation Science and Engineering, 1(1):7383, 2004.

[14] S.R. Searle, G. Casella, and C.E. McCulloch. Variance components, volume 391. John Wiley \& Sons, 2009.

[15] Y. Ding, S. Zhou, and Y. Chen. A comparison of process variation estimators for in-process dimensional measurements and control. Journal of Dynamic Systems, Measurement, and Control, 127:69, 2005.

[16] D.W. Apley and J. Shi. Diagnosis of multiple fixture faults in panel assembly. Journal of Manufacturing Science and Engineering, 120(4):793-801, 1998.

[17] P. Stoica and A. Nehorai. On the concentrated stochastic likelihood function in array signal processing. Circuits, Systems and Signal Processing, 14(5):669-674, 1995.

[18] Y. Ding, J. Shi, and D. Ceglarek. Diagnosability analysis of multi-station manufacturing processes. Journal of Dynamic Systems, Measurement, and Control, 124(1):1-13, 2002.

[19] H. d'Assumpcao. Some new signal processors for arrays of sensors. IEEE Transactions on Information Theory, 26(4):441-453, 1980.

[20] W. Kitagawa, G.and Gersch. Smoothness priors analysis of time series, volume 116. Springer Science \& Business Media, 2012.

[21] R.S. Liptser and A.N. Shiryaev. Statistics of random Processes: I. general Theory, volume 5. Springer Science \& Business Media, 2013.

[22] S. Zhou, Y. Ding, Y. Chen, and J. Shi. Diagnosability study of multistage manufacturing processes based on linear mixed-effects models. Technometrics, 2003.

[23] S.X. Ding. Model-based fault diagnosis techniques: design schemes, algorithms, and tools. Springer Science \& Business Media, 2008.

[24] C.W. Helstrom. Statistical Theory of Signal Detection: International Series of Monographs in Electronics and Instrumentation, volume 9. Elsevier, 2013.

[25] D. Koenig and S. Mammar. Design of proportional-integral observer for unknown input descriptor systems. IEEE transactions on automatic control, 47(12):2057-2062, 2002.

[26] A. Khedher, K. Benothman, D. Maquin, and M. Benrejeb. State and sensor faults estimation via a proportional integral observer. In 6th International Multi-Conference on Systems, Signals and Devices, 2009, pages 1-6. IEEE, 2009.

[27] E. Sales-Setien, I. Peñarrocha, D. Dolz, and R. Sanchis. Performancebased design of PI observers for fault diagnosis in LTI systems under gaussian noises. In 3rd Conference on Control and Fault-Tolerant Systems (SysTol), 2016, pages 407-412. IEEE, 2016.

[28] A. Wu, G. Feng, and G. Duan. Proportional multiple-integral observer design for discrete-time descriptor linear systems. International Journal of Systems Science, 43(8):1492-1503, 2012.

[29] D. Koenig. Unknown input proportional multiple-integral observer design for linear descriptor systems: application to state and fault estimation. IEEE Transactions on Automatic Control, 50(2):212-217, 2005.

[30] A. Wu, G. Duan, and W. Liu. Proportional multiple-integral observer design for continuous-time descriptor linear systems. Asian Journal of Control, 14(2):476-488, 2012.

[31] M.S. Grewal. Kalman filtering. In International Encyclopedia of Statistical Science, pages 705-708. Springer, 2011.

[32] C.K. Chui and G. Chen. Kalman filtering with real time applications. Springer, 1999.

[33] L. Ljung. System identification. Springer, 1998.

[34] G.C. Goodwin, S.F. Graebe, and M.E. Salgado. Control system design. Upper Saddle River, page 13, 2001.

[35] J. Rice. Mathematical statistics and data analysis. Nelson Education, 2006.
[36] M. Weber. A weighted central limit theorem. Statistics \& Probability Letters, 76(14):1482-1487, 2006.

[37] M. Pirhooshyaran and S.T.A. Niaki. A double-max mewma scheme for simultaneous monitoring and fault isolation of multivariate multistage auto-correlated processes based on novel reduced-dimension statistics. Journal of Process Control, 29:11-22, 2015.

[38] Y. Ding, P. Kim, D. Ceglarek, and J. Jin. Optimal sensor distribution for variation diagnosis in multistation assembly processes. IEEE Transactions on Robotics and Automation, 19(4):543-556, 2003.

[39] J. Milisavljevic, S. Commuri, J.K. Allen, and F. Mistree. A concurrent design exploration method for realizing networked manufacturing systems. In ASME 2017 International Design Engineering Technical Conferences and Computers and Information in Engineering Conference.

[40] S.R. Searle and A.I. Khuri. Matrix algebra useful for statistics. John Wiley \& Sons, 2017

[41] R.C. Dorf and R.H. Bishop. Modern control systems. Pearson, 2011.

[42] K.B. Petersen, M.S. Pedersen, et al. The matrix cookbook. Technical University of Denmark, 7(15):510, 2008.

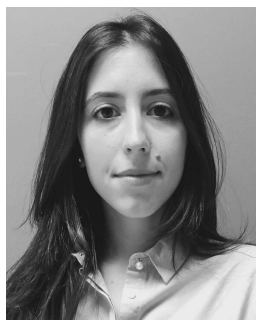

Ester Sales-Setién was born in Castelló, Spain in 1991. She received the MSc degree in Industrial Engineering from the Universitat Jaume I de Castelló and the MSc degree in Mechanical Engineering from Institut National des Sciences Appliquées de Lyon (INSA) in 2014. She received the MSc degree in Energy Efficiency and Sustainability from the Universitat Jaume I de Castelló in 2015. Currently, she is with the Department of Industrial Engineering and Design at the Universitat Jaume I de Castelló, pursuing a $\mathrm{PhD}$ degree with a pre-doctoral grant from the Spanish Government. Her research interests include fault diagnosis and fault tolerant control.

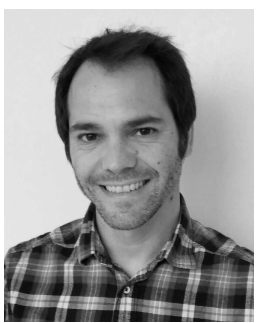

Ignacio Peñarrocha-Alós was born in Castelló, Spain in 1978. He received the M.Sc. degree in Industrial Engineering from the Universitat Jaume I de Castelló in 2002, and his Ph.D. in Computering and Control Engineering from Universitat Politécnica de Valéncia (UPV) in 2006. He has been working since 2004 at the Universitat Jaume I de Castelló. His current position is as an associate professor at the Department of Industrial Engineering and Design. $\mathrm{He}$ has participated in several local and national research projects. His research interests include identification, estimation, fault diagnosis and control over networks, and fault tolerant control of wind energy systems.

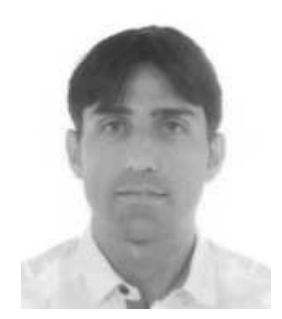

José V. Abellán-Nebot is an Assistant Professor in the Department of Industrial Engineering and Design at Universitat Jaume I de Castelló, Spain. He received his M.S. in Industrial Engineering and his $\mathrm{Ph} . \mathrm{D}$. in Technological Innovation Projects in Product and Process Engineering from the Universitat Jaume I de Castelló in 2003 and 2011, respectively. He was a Visiting Scholar at Monterrey Institute of Technology (2005), University of Michigan (2007), University of Arizona (2009 and 2012) and Georgia Institute of Technology (2014). His research interests focus on product quality due to the stream of variation in manufacturing systems and intelligent machining systems. 